УДК 004.942

ISSN 1995-5499

DOI: https://doi.org/10.17308/sait.2020.2/2916

Поступила в редакцию 30.04.2020

Подписана в печать 15.06.2020

\title{
ВЫБОР ВАРИАНТОВ ФУНКЦИОНИРОВАНИЯ ГЕТЕРОГЕННЫХ СИСТЕМ БЕЗОПАСНОСТИ
}

\author{
(c) 2020 В. В. Меньших ${ }^{\bowtie}$, Н. Е. Спиридонова
}

\author{
Воронежский институт МВД России \\ пр-т Патриотов, 53, 394065 Воронеж, Российская Федерация
}

\begin{abstract}
Аннотация. В работе рассматривается гетерогенная система безопасности, элементами которой являются силы и средства взаимодействующих между собой правоохранительных органов. Основная задача для данной системы состоит в раскрытии, предупреждении и пресечении преступлений, в том числе преступлений против общественной безопасности и общественного порядка. Для решения указанной задачи правоохранительными органами осуществляется мониторинг оперативной обстановки в регионе, а также контроль за различными типами объектов. Однако в случае возникновения чрезвычайных ситуаций, массовых беспорядков и/или других дестабилизирующих факторов, текущее распределение сил и средств может оказаться неэффективным. В этом случае необходим поиск наиболее оптимального распределения имеющихся сил и средств по охраняемым объектам, с учетом сложившейся обстановки в конкретном регионе.

С этой целью осуществляется формализация задачи, включающая описание параметров, влияющих на оценку эффективности функционирования гетерогенной системы безопасности. На этой основе были получены модель оценки защищенности объектов в зависимости от выбора варианта обеспечения безопасности и модель оптимизации выбора вариантов функционирования гетерогенной системы безопасности. Для вариантов функционирования гетерогенной системы безопасности предусмотрено выполнение условия максимальной защищенности всех объектов, подлежащих охране.

Разработанная модель представляет собой задачу нелинейного булева программирования большой размерности, что не позволяет использовать для нахождения решения точные методы. В связи с этим разработан численный метод решения поставленной задачи, основанный на принципе «жадных» алгоритмов и учитывающий особенности предметной области.
\end{abstract}

Ключевые слова: безопасность, гетерогенная система, оценка защищенности, оптимизационная модель, распределение сил и средств.

\section{ВВЕДЕНИЕ}

В современных условиях при борьбе с преступностью необходимо активное взаимодействие подразделений полиции и других правоохранительных органов. В этих целях в субъектах Российской Федерации разрабатываются планы единой дислокации, которые включают в себя описание границ и характеристику обслуживаемой территории, наличие объектов транспортной инфраструктуры,

Меньших Валерий Владимирович e-mail: menshikh@list.ru особо важных и режимных объектов; а также информацию о силах и средствах, участвующих в обеспечении правопорядка, перечень постов и маршрутов патрулирования нарядов полиции, сотрудников (военнослужащих) Росгвардии, и иную необходимую информацию [1]. При этом немаловажная роль в решении задач обеспечения общественного порядка (ООП) отводится обязательному применению сотрудниками правоохранительных органов современных технических средств.

На сегодняшний день существует множество программных, аппаратных, а также программно-аппаратных продуктов, призванных Контент доступен под лицензией Creative Commons Attribution 4.0 License.

The content is available under Creative Commons Attribution 4.0 License. 


\section{В. В. Меньших, Н. Е. Спиридонова}

обеспечивать безопасность в той или иной сфере $[2,3]$. Применение сотрудниками правоохранительных органов таких технических средств способствует появлению своего рода синергетических эффектов, приводящих к повышению эффективности борьбы с преступностью. Так, во многих городах успешно функционируют аппаратно-программные комплексы «Безопасный город» [4]. Благодаря такой системе городского видеонаблюдения раскрывается более половины преступлений против общественного порядка, и процент раскрываемости продолжает неуклонно расти [5].

Перечисленные силы и средства обеспечения безопасности (ССОБ) представляют собой единую систему, которая носит гетерогенный характер, так как входящие в нее объекты различны по своим характеристикам и выполняемым функциям.

Однако в виду ограниченности имеющихся технических ресурсов, не представляется возможным обеспечить постоянный визуальный контроль за всеми типами городских объектов, среди которых: объекты критической инфраструктуры, топливно-энергетического комплекса, социального и культурного назначения и т. д. В связи с этим, необходимо разработать модель оптимизации функционирования гетерогенной системы безопасности, которая позволила бы реорганизовать систему таким образом, чтобы все объекты, упорядоченные по степени важности, максимально возможное время находились под наблюдением. Вопросами оптимального распределения сил и средств по объектам охраны занимались многие исследователи [6-10]. Решению этой задачи также частично посвящены работы $[11,12]$, однако они не учитывают наличия в гетерогенных системах безопасности подвижных элементов.

\section{МАТЕРИАЛЫ И МЕТОДЫ}

Формализация задачи. Обратимся к описанию системы обеспечения общественной безопасности на примере города. При расстановке сил и средств данной системы учитываются следующие основные факторы:
- особенности конкретного региона, включающие в себя расположение, социально-политическую обстановку и т. д.;

- места размещения и режим работы объектов критической инфраструктуры, жизнеобеспечения, объектов социального назначения, банков и т. д.;

- динамика и структура преступлений, совершаемых на улицах, площадях, скверах;

- метеорологические условия, а также время суток.

В работах $[13,14]$ исследуются системы безопасности, включающие в себя подвижные или стационарные объекты в отдельности. В рамках данной работы рассмотрим систему, которая состоит из следующих подсистем разнородных объектов:

1. Подсистема стаиионарных сил и средств. Данная подсистема включает в себя посты различных типов (пост по охране объекта, пост по охране определенной территории), а также технические средства видеонаблюдения (поворотные и бесповоротные видеокамеры). Управление системой видеонаблюдения осуществляется с помощью ситуационного центра (СЦ) [15].

2. Подсистема подвижных сил и средств. В качестве подвижных объектов будем рассматривать пешие и автомобильные патрули, имеющие различные протяженности маршрутов.

Так как обязанности по обеспечению общественного правопорядка возлагаются не только на сотрудников полиции, но и на другие правоохранительные органы, необходим межведомственный координационный центр (МКЦ), который позволил бы обеспечить их эффективное взаимодействие. Схема организационного взаимодействия в такой системе представлена на рис. 1.

Введем следующие обозначения:

- $B_{1}$ - множество типов камер;

- $B_{2}$ - множество типов постов;

- $B_{3}$ - множество маршрутов патрулей;

- $B=B_{1} \cup B_{2} \cup B_{3}$;

- $r_{j k}^{1}$ - степень защищенности объекта $o_{j}$ при выборе камеры $k$-го типа;

- $r_{j k}^{2}$ - степень защищенности объекта $o_{j}$ при выборе поста $k$-го типа; 
- $r_{j k}^{3}$ - степень защищенности объекта $o_{j}$ при выборе $k$-го маршрута.

Примем, что $r_{j k}^{i} \in[0,1]$ и задаются экспертами с учётом результатов анализа оперативной обстановки и требований руководящих документов или рассчитываются на основе использования известных математических моделей $[16,17]$.

Будем считать заданным множество охраняемых объектов $O=\left\{o_{1}, o_{2} \ldots o_{\mid \rho}\right\}$ и оценки их важности $A=\left\{\alpha_{1}, \alpha_{2} \ldots \alpha_{|O|}\right\}$ (они могут быть определены на основе использования метода анализа иерархий [18], в этом случае $\left.\sum_{i=1}^{|O|} \alpha_{i}=1\right)$, а также множество вариантов обеспечения безопасности этих объектов $V$. Каждый вариант $v \in V$ обеспечивает достижение определенной степени защищенности $P(v)$, которая зависит от защищенности каждого объекта в отдельности и от его важности:

$$
P(v)=\sum_{j=1}^{|O|} \alpha_{j} s_{j}(v),
$$

где $s_{j}(v)$ - степень защищенности объекта $o_{j}$ при реализации для него варианта обеспечения безопасности $v \in V$.

Задача состоит в том, чтобы найти такой вариант обеспечения безопасности $v^{*}$, который обеспечивал бы максимизацию целевой функции (1), т. е.

$$
v^{*}=\operatorname{Arg} \max P(v)=\underset{v \in V}{\operatorname{Arg} \max } \sum_{j=1}^{|O|} \alpha_{j} s_{j}(v) .
$$

Для решения указанной задачи необходимо разработать модель оценки защищенно- сти объектов $s_{j}(v)$ в зависимости от выбора варианта обеспечения безопасности.

Выбор вариантов может быть описан следующими переменными:

$x_{j k}^{1}=\left\{\begin{array}{l}1, \text { если для обеспечения охраны объекта } \\ \text { используется камера } k \text {-го типа, } \\ 0, \text { если иначе. }\end{array}\right.$ $x_{j k}^{2}=\left\{\begin{array}{l}1, \text { если для обеспечения охраны объекта } \\ \text { используется пост } k \text {-го типа, } \\ 0, \text { если иначе. }\end{array}\right.$ $x_{j k}^{3}=\left\{\begin{array}{l}1, \text { если для обеспечения охраны объекта } \\ \text { используется } k \text {-й маршрут, } \\ 0, \text { если иначе. }\end{array}\right.$

Таким образом, вариант охраны $v \in V$ описывается набором значений переменных $X=\left(x_{j k}^{1}, x_{j k}^{2}, x_{j k}^{3}\right)$. Вариант является допустимым, если выполняются следующие условия:

$$
\begin{aligned}
& \sum_{j=1}^{|O|} x_{j k}^{1} \leq n_{k}^{1}, k=1, \ldots,\left|B_{1}\right|, \\
& \sum_{j=1}^{|O|} x_{j k}^{2} \leq n_{k}^{2}, k=1, \ldots,\left|B_{2}\right|, \\
& \sum_{j=1}^{|O|} x_{j k}^{3} \leq n_{k}^{3}, k=1, \ldots,\left|B_{3}\right|,
\end{aligned}
$$

где $n_{k}^{1}-$ количество имеющихся камер $k$-го типа, $n_{k}^{2}-$ количество имеющихся постов $k$-го типа, $n_{k}^{3}-$ число патрулей. Кроме того, для каждого объекта $o_{j}$ должно выполняться требование:

$$
\sum_{s=1}^{3} \sum_{k=1}^{n_{k}^{s}} x_{j k}^{s} \geq 1
$$

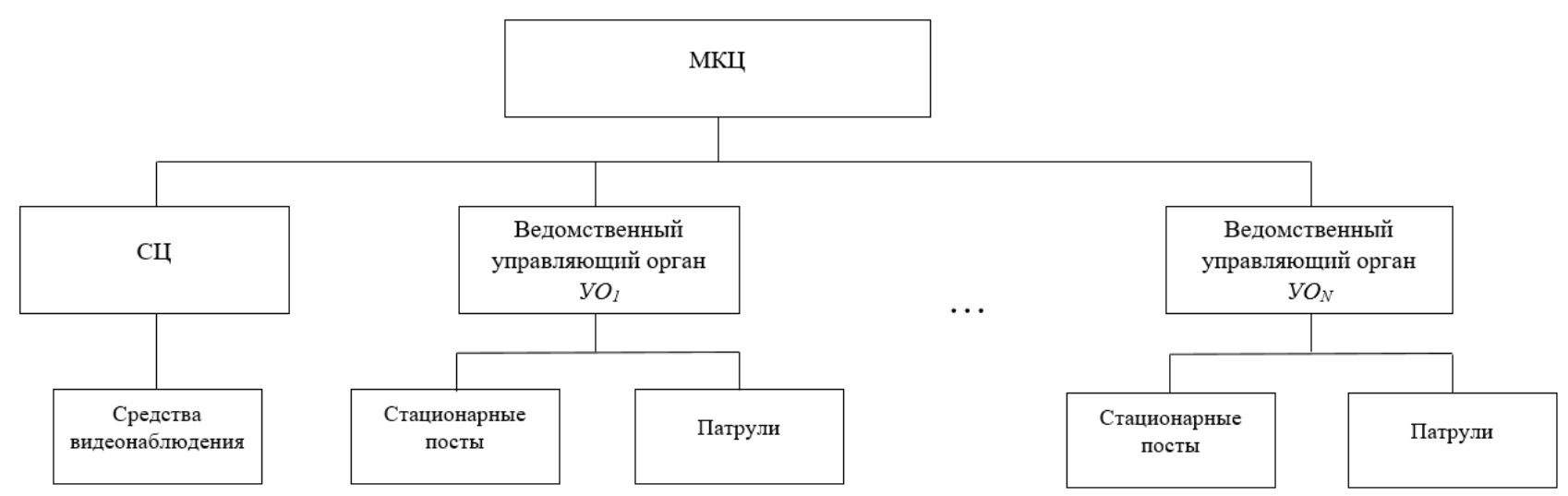

Рис. 1. Обобщенная схема организации обеспечения безопасности города [Fig. 1. General scheme for a city's security system] 


\section{В. В. Меньших, Н. Е. Спиридонова}

означающее, что объект защищён хотя бы одним средством.

Выбор варианта защиты позволяет дать оценку защищенности объектов каждым типом средств: $r_{j k}^{1} x_{j k}^{1}-$ защищенность объекта при выборе камеры $k$-го типа; $r_{j k}^{2} x_{j k}^{2}-$ защищенность объекта при выборе поста $k$-го типа; $r_{j k}^{3} x_{j k}^{3}$ - защищенность объекта при выборе $k$-го маршрута.

Учитывая, что понятие «степень защищенности» близко к понятию «вероятность защищенности» для оценки $s_{j}$ могут быть использованы формулы, аналогичные формулам теории вероятности.

Возможности защиты одного объекта различными средствами можно считать независимыми событиями. Во избежание громоздкости введем новые переменные, функционально связанные со старыми переменными следующим образом:

$$
y_{j k}=r_{j k}^{1} x_{j k}^{1} ; y_{j k+\left|B_{1}\right|}=r_{j k}^{2} x_{j k}^{2} ; y_{j k+\left|B_{1}\right|+\left|B_{2}\right|}=r_{j k}^{3} x_{j k}^{3} .
$$

Тогда защищенность объекта $o_{j}$ с помощью всех средств обеспечения безопасности в соответствии с вариантом $v \in V$ определяется по формуле (7):

$$
\begin{gathered}
s_{j}(v)=\sum_{q=1}^{|B|} y_{j q}-\sum_{q_{1}, q_{2}=1}^{|B|} y_{j q_{1}} y_{j q_{2}}+ \\
+\sum_{q_{1}, q_{2}, q_{3}=1}^{B \mid} y_{j q_{1}} y_{j q_{2}} y_{j q_{3}}+\ldots \\
\ldots+(-1)^{|B|-1} \sum_{q_{1}, \ldots, q_{|B|} \mid=1}^{|B|} y_{j q_{1}} \ldots y_{j q_{|B|}} .
\end{gathered}
$$

Задача (2)-(6) с учётом явного вида функции защищённости (7) представляет собой задачу нелинейного булева программирования большой размерности, использование точных методов для решения которой практически невозможно. Поэтому обратимся к разработке численного метода её решения с учётом описанных выше особенностей взаимосвязей между переменными и параметрами.

Описание численного метода. Учитывая высокую размерность задачи целесообразно использовать принцип «жадных» алгоритмов, основанных на идеях локальной оптимизации и использующих итерационный процесс последовательного нахождения решения. При- менительно к условиям решаемой задачи это означает, что распределение средств обеспечения безопасности и, следовательно, объектов, на которые они распределены, осуществляется последовательно.

Представим всё множество средств обеспечения безопасности в следующем виде: $B=B^{I} \cup B^{I I}$, где $B^{I}-$ множество распределённых средств; $B^{I I}=B \backslash B^{I}$ - множество нераспределённых средств.

Тогда оценка эффективности частичного решения, заключающегося в распределении только части средств, находится по формуле (8):

$$
\sum_{j=1}^{O Q} \alpha_{j} s_{j}\left(B^{I}\right)
$$

где $s_{j}\left(B^{I}\right)$ - защищенность всех объектов средствами из множества $B^{I}$. Эта величина может быть найдена по формуле (9):

$$
\begin{gathered}
s_{j}\left(B^{I}\right)=\sum_{q=1}^{|B|} z_{j q}-\sum_{q_{1}, q_{2}=1}^{|B|} z_{j q_{1}} z_{j q_{2}}+ \\
+\sum_{q_{1}, q_{2}, q_{3}=1}^{|B|} z_{j q_{1}} z_{j q_{2}} z_{j q_{3}}+\ldots \\
\ldots+(-1)^{|B|-1} \sum_{q_{1}, \ldots, q_{|| B \mid}=1}^{|B|} z_{j q_{1}} \ldots z_{j q_{|B|}}, \\
\text { где } z_{j q}=y_{j q} \delta_{q}, \delta_{q}=\left\{\begin{array}{l}
1, \text { если } b_{q} \in B^{I}, \\
0, \text { если } b_{q} \in B^{I I} .
\end{array}\right.
\end{gathered}
$$

Аналогично множеству средств обеспечения безопасности, представим всё множество объектов в виде $O=O^{I} \cup O^{I I}$, где

$O^{I}$ - множество объектов, на которые распределены средства;

$O^{I I}=O \backslash O^{I}$ - множество объектов, на которые не распределены средства.

Введем следующие обозначения:

$O(b)$ - множество объектов, безопасность которых обеспечивается средством $b$;

$$
w(b, o)=\left\{\begin{array}{l}
1, \text { если } b \text { назначен на объект } o, \\
0, \text { если иначе; }
\end{array}\right.
$$

$W=(w(b, o)), b \in B, \quad o \in O-$ матрица, задающая распределение средств обеспечения безопасности по объектам, т. е. некоторый вариант $v \in V$.

Особенность решаемой задачи в том, что в процессе оптимизации требуется выполнение двух условий: 
- во-первых, распределение ССОБ на все охраняемые объекты, т.е. выполнение условия (6);

- во-вторых, обеспечение максимальной безопасности всей системы в целом.

Укрупнённая блок-схема алгоритма распределения средств обеспечения безопасности по объектам представлена на рис. 2-3.

Приведём описание отдельных блоков данной блок-схемы.

1. Ввод начальных значений. Первоначально будем считать, что множество распределённых ССОБ, а также множество защищённых объектов пусты: $B^{I}=\varnothing, B^{I I}=B, O^{I}=\varnothing$, $O^{I I}=O ; W=Q$ (здесь $Q-$ обозначение нулевой матрицы).
Далее будем осуществлять итерации, на каждой из которых выбирается средство, в максимальной степени сокращающее мощность множества $O^{I I}$. Заметим, что после назначения средства на объекты, возможности остальных ССОБ во множестве $B^{I I}$ по обеспечению безопасности объектов из множества $O^{I I}$ изменяются. Это требует оценки их возможностей на каждой итерации. Вместе с тем, вновь добавляемые ССОБ могут увеличить защищенность ранее включенных в $O^{I}$ объектов.

2. Нахождение множества средств с наибольшим охватом объектов. Для выполнения условия (6), требующего обязательной охраны всех объектов, ранжируем все ССОБ, не-

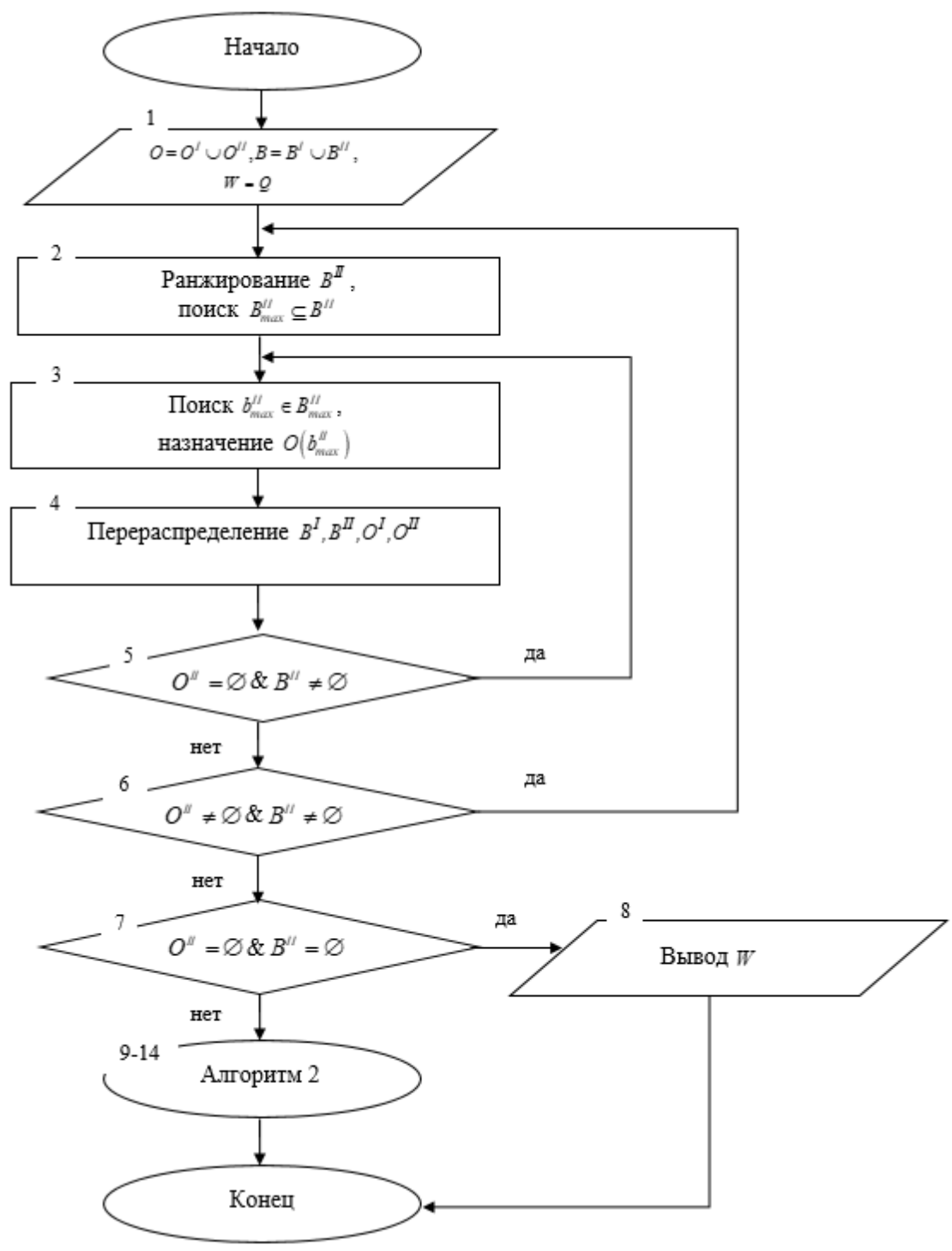

Рис. 2. Блок-схема распределения средств обеспечения безопасности по объектам

[Fig. 2. Flowchart for the allocation of manpower and resources to protected locations (part 1)] 


\section{В. В. Меньших, Н. Е. Спиридонова}

зависимо от их типа, по количеству охраняемых объектов множества $O^{I I}$. После этого выделим множество $B_{\max }^{I I} \subseteq B^{I I}$ средств, обеспечивающих охрану наибольшего количества нераспределённых объектов.

3. Выбор и назначение средства, максимизирующего частичную оценку. Найдем $b_{\max }^{I I} \in B_{\max }^{I I}$, который максимизирует оценку эффективности частичного решения, т. е. величину $\sum_{j=1}^{|O|} \alpha_{j} s_{j}\left(B^{I} \cup\left\{b_{\max }^{I I}\right\}\right)$. Будем считать, что средство $b_{\max }^{I I}$ назначено на объекты множества $O\left(b_{\max }^{I I}\right)$, т. е. $\forall o \in O\left(b_{\max }^{I I}\right) w\left(b_{\max }^{I I}, o\right)=1$.

Если обеспечена охрана всех объектов, т. е. $O^{I I}=\varnothing$ и назначены все ССОБ, т. е. $B^{I I}=\varnothing$, то получено решение задачи и осуществляется вывод результатов.

Если остались незащищённые объекты, т. е. $O^{I I} \neq \varnothing$, но нет свободных средств обеспечения безопасности, т. е. $B^{I I}=\varnothing$, то в таком случае перераспределяем (ССОБ) в соответствии с алгоритмом 2 (для этого осуществляем переход к блоку 6).

8. Вывод результата. Результатом решения задачи является матрица $W$.

9. Нахождение дублированных средств обеспечения безопасности. Если израсходованы все средства, т. е. $B^{I I}=\varnothing$, но защищены не все объекты, т. е. $O^{I I} \neq \varnothing$, то обратимся к нахождению множества полностью дублированных средств обеспечения безопасности $B^{d} \subseteq B^{I}=B$ (средство $b_{i}$ полностью дублировано средством $b_{j}$, если $\left.\forall o \in O w\left(b_{i}, o\right) \leq w\left(b_{j}, o\right)\right)$.

10. Проверка условия. Если все средства $B^{d}$ исчерпаны, т. е. $B^{d}=\varnothing$, но остались незащищенные объекты, т. е. $O^{I I} \neq \varnothing$, то при заданных условиях силы и средства не могут обеспечить охрану всех объектов, и осуществляется переход к блоку 11, иначе переход к блоку 12.

12. Нахождение множества дублированных средств с наибольшим охватом объектов. Первоначально ранжируем все средства $B^{d}$ по количеству охраняемых объектов множества $O^{I I}$. После этого выделим множество $B_{\text {max }}^{d} \subseteq B^{d}$ средств, обеспечивающих охрану наибольшего количества нераспределённых объектов.

13. Выбор и назначение средства, максимизирующего частичную оценку. Найдем $b_{\max }^{d} \in B_{\max }^{d}$, максимизирующего величину $\sum_{j=1}^{|0|} \alpha_{j} s_{j}\left(B^{I} \cup\left\{b_{\max }^{d}\right\}\right)$. После этого перераспределим элементы множества объектов

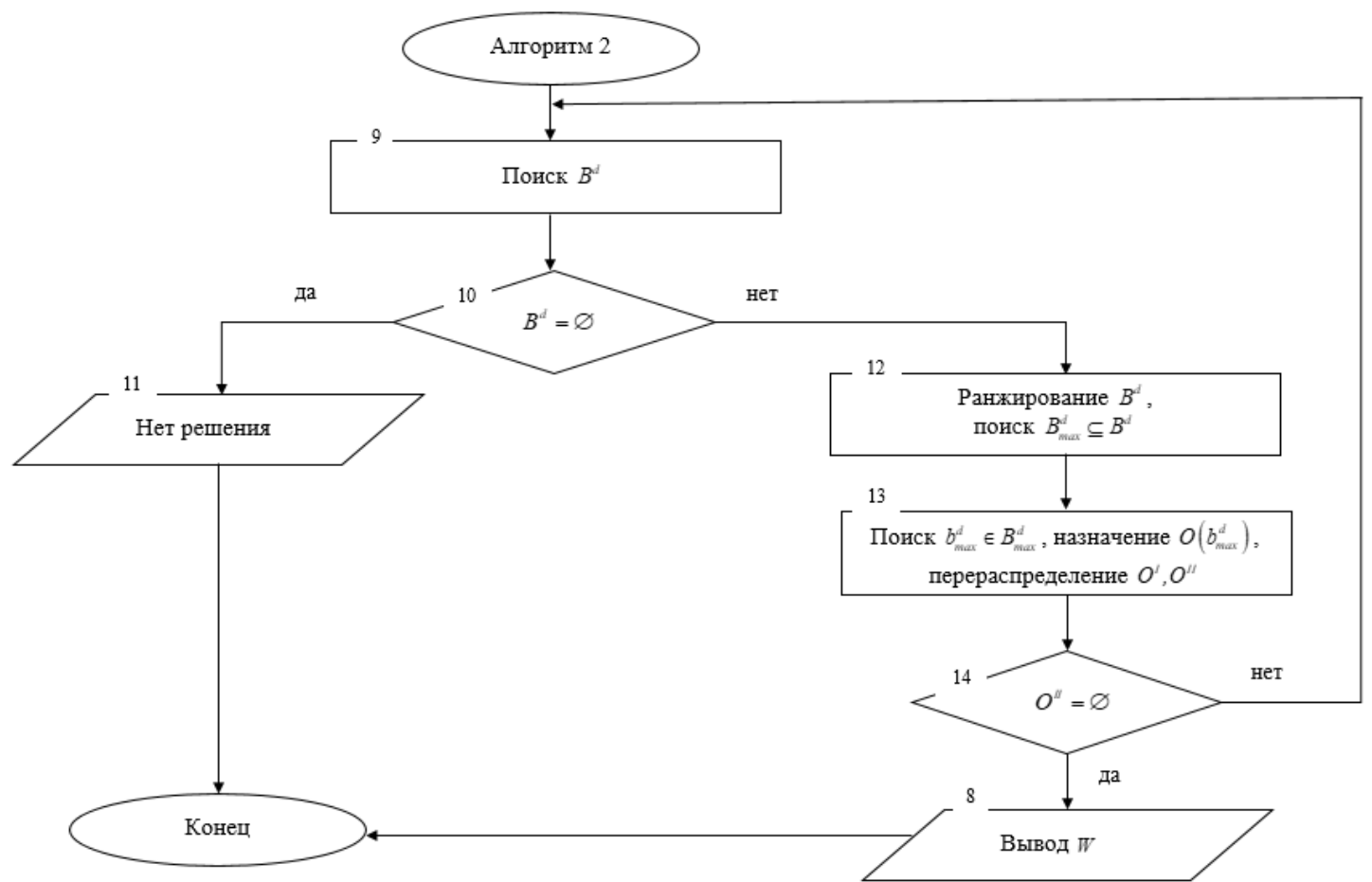

Рис. 3. Блок-схема распределения средств обеспечения безопасности по объектам (часть 2)

[Fig. 3. Flowchart for the allocation of manpower and resources to protected locations (part 2)] 
следующим образом: $O^{I}=O^{I} \cup O\left(b_{\max }^{d}\right)$, $O^{I I}=O^{I I} \backslash \mathrm{O}\left(b_{\max }^{d}\right)$.

14. Проверка условия. Итерации продолжаются до исчерпания всех незащищенных объектов, т. е. до тех пор пока $O^{I I} \neq \varnothing$.

\section{ЗАКЛЮЧЕНИЕ}

Задача выбора оптимального варианта распределения сил и средств по охраняемым объектам (2)-(6) содержит следующие параметры:

$$
\begin{gathered}
\alpha_{j}, \mathrm{j}=1, \ldots,|O|, \\
r_{j k}^{s}, s=1,2,3, j=1, \ldots,|O|, b=1, \ldots \mathrm{n}_{k}^{s} .
\end{gathered}
$$

Как указано выше, для получения численных оценок данных параметров целесообразно использовать методы экспертного оценивания, поскольку репрезентативные статистические данные, как правило, отсутствуют. В связи с этим, при решении данной задачи нет необходимости в применении точных методов оптимизации, поскольку они не смогут существенно повысить обоснованность принимаемых решений по размещению ССОБ.

Полученная модель имеет универсальный характер, и может быть применена как для распределения сил и средств конкретного ведомства, так и для распределения сил и средств в рамках системы единой дислокации правоохранительных органов.

\section{КОНФЛИКТ ИНТЕРЕСОВ}

Авторы декларируют отсутствие явных и потенциальных конфликтов интересов, связанных с публикацией настоящей статьи.

\section{СПИСОК ЛИТЕРАТУРЫ}

1. Об утверждении Наставления об организации в органах внутренних дел Российской Федерации деятельности по обеспечению правопорядка на улицах и в иных общественных местах : приказ МВД России от 25.11.2019 г. № 879.

2. Han, Hoon. Smart CCTV and the Management of Urban Space. / Han Hoon \& Hawken, Scott \& Williams, Angelique // Handbook of Re- search on Digital Media and Creative Technologies. - 2015. - P. 430-447.

3. Ляликова В. Г. Система обнаружения и сохранения лица человека в кадре / В. Г. Ляликова, М. М. Безрядин, А. К. Шатаев // Вестник Воронеж. гос. ун-та. Сер. Системный анализ и информационные технологии. - 2017. -№ 3. C. $110-114$.

4. Об утверждении Концепции построения и развития аппаратно-программного комплекса «Безопасный город» : Распоряжение Правительства Российской Федерации от 03.12.2014 г. № 2446-p.

5. Сведения о состоянии преступности в Российской Федерации за январь - декабрь 2019 года : [Электронный ресурс] // аналитический отчет ФКУ ГИАЦ МВД России. - Режим доступа: https://мвд.pф/reports/ item/19412450/ (Дата обращения: 01.03.2020г.)

6. Huanfa Chen. Designing efficient and balanced police patrol districts on an urban street network / Huanfa Chen, Tao Cheng, Xinyue Ye // International Journal of Geographical Information Science. - 2019. - P. 269-290.

7. Zhang Y. Police patrol districting method and simulation evaluation using agent-based model \& GIS / Y. Zhang, D. E. Brown // Secur Inform. - 2013.

8. Berestycki H., Nadal J. Self-organised critical hot spots of criminal activity. // European Journal of Applied Mathematics. - 2010. - P. 371-399.

9. Joan Saldana, Maria Aguareles, Albert Avinyó, Marta Pellicer, Jordi Ripoll. An Age-Structured Population Approach for the Mathematical Modeling of Urban Burglaries // SIAM Journal on Applied Dynamical Systems. - 2018.

10. Lacey A., Tsardakas M. A mathematical model of serious and minor criminal activity // European Journal of Applied Mathematics. 2016. - P. 403-421.

11. Menshikh V. V. Model of optimization of arrangement of video surveillance means with regard to ensuring their own security / V. V. Menshikh, D. Iu. Kalkov, N. E. Spiridonova // 1st International Conference on Control Systems, Mathematical Modelling, Automation and Energy Efficiency (SUMMA). - 2019. - P. 4-7. 


\section{В. В. Меньших, Н. Е. Спиридонова}

12. Menshikh V. V. Structural-parametric modeling of heterogeneous systems for ensuring their safety / V. V. Menshikh, N. E. Spiridonova // XXI International Conference Complex Systems: Control and Modeling Problems (CSCMP). 2019. - P. 159-162.

13. Меньших B. В. Оптимизация распределения групп реагирования по объектам защиты / В. В. Меньших, Д. Ю. Калков // Информационная безопасность регионов. 2014. - № 4(17). - С. 47-54.

14. Меньших B. В. Алгоритм оптимизации контроля безопасности дорожного движения / В. В. Меньших, П. В. Орехов // Приборы и системы. Управление, контроль, диагностика. - 2015. - № 5. - С. 30-35.

15. Меньших В. В. Использование ситуационных центров для обучения действиям в кризисных ситуациях / В. В. Меньших, О. В. Пьянков, А. Ф. Самороковский // Ин- формационная безопасность регионов. 2011. - № 2(9). - С. 104-107.

16. Калков Д. Ю. Модели и алгоритмы оптимизации порядка проверки охраняемых объектов при получении сигналов тревоги : автор. дис. ...канд. техн. наук : 05.13.18 / Калков Дмитрий Юрьевич. -Воронеж, 2016. -16с.

17. Дурденко В. А. Моделирование и оценка эффективности интегрированных систем безопасности объектов, подлежащих обязательной государственной охране / В. А. Дурденко, А. А. Рогожин, Б. О. Баторов // Вестник Воронеж. гос. ун-та. Сер. Системный анализ и информационные технологии. - 2019. № 4. -С. 82-92.

18. Саати T. Принятие решений. Метод анализа иерархий / Т. Саати ; пер. с англ. Р. Г. Вачнадзе. - Москва : Радио и связь. 1993. -278 c.

Меньших Валерий Владимирович - д-р физ.-мат. наук, проф., профессор кафедры математики и моделирования систем, Воронежский институт МВД России.

E-mail: menshikh@list.ru

ORCID iD: https://orcid.org/0000-0001-9235-4997

Спиридонова Наталья Евгеньевна - адъюнкт, Воронежский институт МВД России. E-mail: spiridonova_n@icloud.com ORCID iD: https://orcid.org/0000-0002-0191-3114 


\title{
THE CHOICE OF OPTIONS FOR HETEROGENEOUS SECURITY SYSTEMS
}

\author{
(c) 2020 V. V. Menshikh ${ }^{\bowtie}$, N. E. Spiridonova \\ Voronezh Institute of the Ministry of the Interior of Russia \\ 53, Patriotov Avenue, 394065 Voronezh, Russian Federation
}

\begin{abstract}
Annotation. The article considers a heterogeneous security system, whose elements are the manpower and resources of the interacting law enforcement agencies. The main functions of this system are the prevention, suppression, and discovery of criminal offences, including crimes against public safety and order. To solve this problem, law enforcement agencies monitor the operational situation in the region, as well as various types of urban facilities. However, in case of emergencies, riots and/or other destabilising factors, the current allocation of manpower and resources may be ineffective. In this case, it is necessary to search for the optimal allocation of available manpower and resources among protected locations, taking into account the current situation in a particular region.

In order to do this, we formalised the task, which included the description of the parameters affecting the assessment of the effectiveness of the functioning of a heterogeneous security system. Based on this, a model was obtained for assessing the security of objects depending on the choice of a security option and a model for optimising the choice of options for the functioning of a heterogeneous security system. All the considered options for the functioning of the heterogeneous security system suggest that the conditions for the maximum security of all objects to be protected are met.

The developed model is a high-dimensional nonlinear Boolean programming problem, which prevented us from using exact methods to find a solution. Therefore, we developed a numerical method for solving the problem, based on the theory of greedy algorithms and taking into account the characteristics of the subject area.
\end{abstract}

Keywords: security, heterogeneous system, security assessment, optimisation model, allocation of manpower and resources.

\section{CONFLICT OF INTEREST}

The authors declare the absence of obvious and potential conflicts of interest related to the publication of this article.

\section{REFERENCES}

1. On approval of the Manual on the organization in the internal affairs bodies of the Russian Federation of activities to ensure law and order on the streets and in other public places: Order of the Ministry of Internal Affairs of Russia dated November 25, 2019. No 879.

2. Hoon Han, Scott Hawken, Angelique Williams. Smart CCTV and the Management of Ur-

Menshikh Valery V.

e-mail: menshikh@list.ru ban Space // Handbook of Research on Digital Media and Creative Technologies. 2015. P. 430447.

3. Lyalikova V. G., Bezryadin M. M., Shataev $A$. K. The system for detecting and saving a person in a frame // Bulletin of the Voronezh State University. Series: System Analysis and Information Technology. 2017. No 3. P. 110-114.

4. On approval of the Concept for the construction and development of the Safe City hardware-software complex: Order of the Government of the Russian Federation dated December 3, 2014. № 446-r.

5. Information on the state of crime in the Russian Federation for January - December 2019: [Electronic resource] // analytical report of the PKU GIATS of the Ministry of Internal Affairs of Russia. Available at: https: //mvd.rf / reports / item / 19412450 (accessed: 03/01/2020) 


\section{B. В. Меньших, Н. Е. Спиридонова}

6. Huanfa Chen, Tao Cheng, Xinyue Ye. Designing efficient and balanced police patrol districts on an urban street network // International Journal of Geographical Information Science. 2019. P. 269-290.

7. Zhang Y., Brown D. E. Police patrol districting method and simulation evaluation using agent-based model \& GIS // Secur Inform. 2013.

8. Berestycki H., Nadal J. Self-organised critical hot spots of criminal activity. // European Journal of Applied Mathematics. 2010. P. 371399.

9. Joan Saldana, Maria Aguareles, Albert Avinyó, Marta Pellicer, Jordi Ripoll. An Age-Structured Population Approach for the Mathematical Modeling of Urban Burglaries // SIAM Journal on Applied Dynamical Systems. 2018.

10. Lacey A., Tsardakas M. A mathematical model of serious and minor criminal activity // European Journal of Applied Mathematics. 2016. P. 403-421.

11. Menshikh V. V., Kalkov D. Iu., Spiridonova N. E. Model of optimization of arrangement of video surveillance means with regard to ensuring their own security // 1st International Conference on Control Systems, Mathematical Modelling, Automation and Energy Efficiency (SUMMA). 2019. P. 4-7.

12. Menshikh V. V., Spiridonova N. E. Structural-parametric modeling of heterogeneous systems for ensuring their safety // XXI Internation- al Conference Complex Systems: Control and Modeling Problems (CSCMP). 2019. P. 159-162.

13. Menshikh V. V., Kalkov D. Yu. Optimization of the distribution of response groups by objects of protection // Information security of the regions. 2014. No 4 (17). P. 47-54.

14. Menshikh V. V., Orekhov P. V. Algorithm for optimizing traffic safety control // Devices and Systems. Management, control, diagnostics. 2015. No 5. P. 30-35.

15. Menshikh V. V., Pyankov O. V., Samorokovsky A. F. The use of situational centers for training in crisis situations // Information Security of the Regions. 2011. No 2 (9). P. 104-107.

16. Kalkov D. Yu. Models and algorithms for optimizing the procedure for checking protected objects when receiving alarms: author. dis. cand. tech. Sciences: 05.13.18. Voronezh, 2016. 16 p.

17. Durdenko V. A., Rogozhin A. A., Batorov B. O. Modeling and evaluating the effectiveness of integrated security systems of objects subject to mandatory state protection // Bulletin of Voronezh State University. Series: System Analysis and Information Technology. 2019. No 4. P. 82-92.

18. Saati T. Decision Making. Hierarchy analysis method; per. from English R. G. Vachnadze. Moscow : Radio and communications, 1993. $278 \mathrm{p}$.

Menshikh Valery V. - DSc in Physics and Mathematics, Professor, Department of Mathematics and Systems Modelling, Voronezh Institute of the Ministry of Internal Affairs of the Russian Federation.

E-mail: menshikh@list.ru

ORCID iD: https://orcid.org/0000-0001-9235-4997

Spiridonova Natalia E. - adjunct, Voronezh Institute of the Ministry of Internal Affairs of the Russian Federation.

E-mail: spiridonova_n@icloud.com

ORCID iD: https://orcid.org/0000-0002-0191-3114 